\title{
STUDY OF SPONTANEOUS POLARISATION AND PYROELECTRIC MODULUS FOR THE TRIGLYCINE SULPHATE CRYSTALS DOPED BY CHROMIUM IONS
}

\author{
J. Dziedzic and B. Stolecki
}

Institute of Physics, Technical University of Wrocław Wybrzeże Wyspiańskiego 27, 53-370 Wrocław, Poland

(Received January 3, 2000; revised version April 7, 2000)

The temperature dependencies of a spontaneous polarisation, $P_{s}$, and pyroelectric modulus, $\gamma$, at different electric field intensities were investigated for the triglycine sulphate crystals doped with the $\mathrm{Cr}^{3+}$ chromium ions. On the base of thermodynamic theory the temperature dependencies of $P_{\mathrm{s}}$ and $\gamma$ at different electric field intensities were estimated. The coefficients of the expansion of the thermodynamic potential were determined. The experimental results were compared with those obtained according to the thermodynamic theory.

PACS numbers: $64.70 . \mathrm{Kb}$

\section{Introduction}

The triglycine sulphate (TGS) crystals doped with the $\mathrm{Cr}^{3+}$ chromium ions are characterised by a remarkable change of the elastic properties in comparison to those of the pure crystals [1]. The author of Ref. [2] considers the doped crystals as the double phases systems. One of the phases consists of the non-disturbed crystal lattice of TGS and the second one of the spherical regions disturbed by the aminopyridine (APd) impurities. In Refs. [2-6] the theory of the longitudinal ultrasonic wave scattering on the non-interacting impurity centres was presented.

The results of the studies on the influence of the electric field intensity on polarisation and pyroelectric coefficient of crystals doped with the $\mathrm{Cr}^{3+}$ chromium ions are not available in literature. Therefore this is a reason that author has decided to undertake such investigations.

\section{Thermodynamic relations}

The Gibbs potential may be assumed in a form

$$
G(T, P, X)=G_{0}(T)+\frac{1}{2} A P^{2}+\frac{1}{4} B P^{4}-E P,
$$


where

$$
A=\frac{T-T_{\mathrm{c}}}{\varepsilon_{0} C} .
$$

On the base of the experimental results the phase transition temperature, the coefficients of the expansion in a series of thermodynamic potential and the Curie-Weiss constant were estimated for the TGS crystals doped with the $\mathrm{Cr}^{3+}$ chromium ions, as being $B=6.5 \times 10^{11} \mathrm{~V} \mathrm{~m} / \mathrm{C}, T_{\mathrm{c}}=326.3 \mathrm{~K}$, $C=3560 \mathrm{~K} \mathrm{C} /(\mathrm{F} \mathrm{V})$.
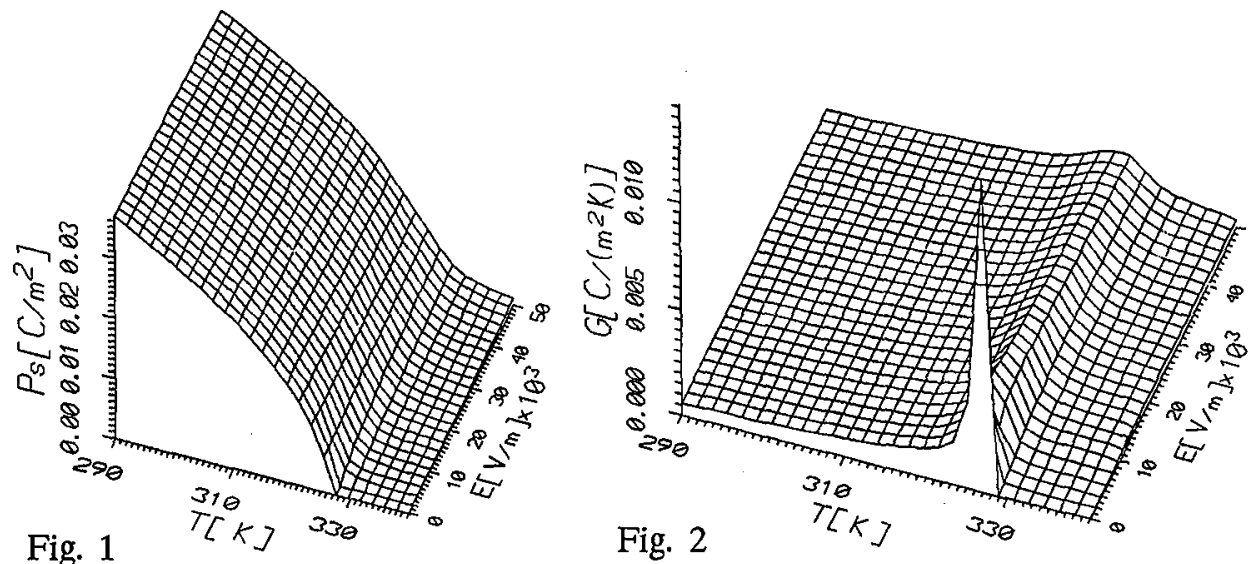

Fig. 1. Temperature dependence of the spontaneous polarisation for several electric field intensities calculated according to the thermodynamic theory.

Fig. 2. Temperature dependence of the pyroelectric modulus for several electric field intensities calculated according to the thermodynamic theory.

The temperature dependencies of the spontaneous polarisation and pyroelectric modulus at different electric field intensity were calculated using the data given above and the thermodynamic relations. The results of calculations are illustrated in Figs. 1 and 2.

\section{Experimental}

The temperature dependencies of the spontaneous polarisation and pyroelectric coefficient at different electric field intensities were investigated by using a method of an integration of the pyroelectric charge when the measurement unit shown in Fig. 3 was applied. Due to application of the negative feedback in the integrating circuit the input resistance was obtained almost equal to zero.

The sample was polarised by using the supplier with a small output resistance, which allowed minimising the charge integration errors connected with the non-zero resistance. The measurement error of the pyroelectric charge does not exceed $1.5 \%$ and that of the pyroelectric current $2.5 \%$.

The measurements were performed for the TGS crystals doped with the $\mathrm{Cr}^{3+}$ chromium ions at concentration of $0.5 \%$ crystallised in the ferroelectric phase. 


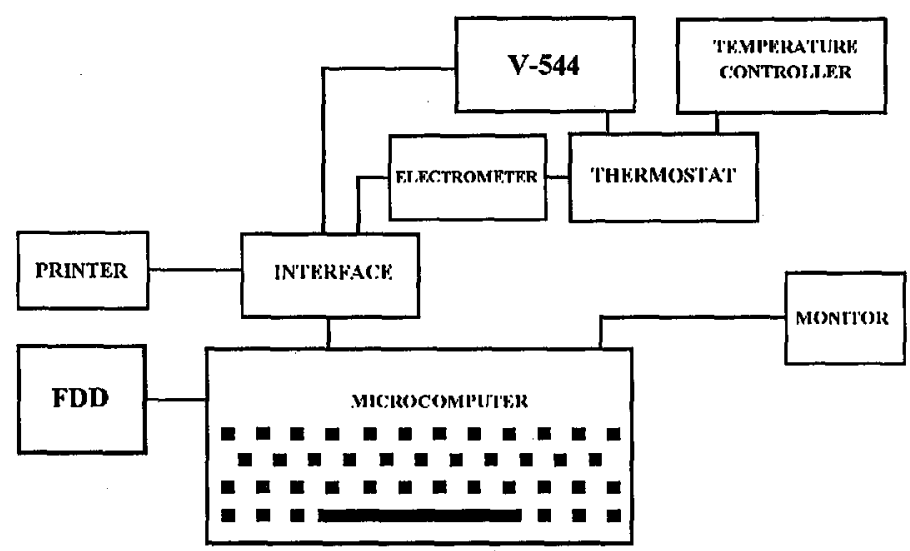

Fig. 3. Block scheme of the system for the measurements of the temperature dependencies of spontaneous polarisation and pyroelectric modulus at different electric field intensities.

The samples for measurements were cut in the disc shape with the flat surface perpendicular to the ferroelectric axis. On the surfaces the silver electrodes were vacuum deposited. Then the copper wires were glued to the electrodes. The wires of the sample were suspended in the thermostat and held at the temperature of $360 \mathrm{~K}$ for $18 \mathrm{~h}$. Immediately after annealing the measurements were performed. All measurements were performed at the constant temperature rate of $1 \mathrm{~K} / \mathrm{min}$.

\section{Results}

The measurements of the spontaneous polarisation and pyroelectric modulus were performed for the TGS crystals doped with the $\mathrm{Cr}^{3+}$ chromium ions at eight different electric field intensity values. The experimental data were elaborated by means of the cringing method and presented in the 3 -dimensional system using the $T-E$ grid.

The experimental results of the spontaneous polarisation $\left(P_{\mathrm{s}}\right)$ are presented in Fig. 4. The curve for the $P_{\mathbf{s}}=f(T)$ dependence at $E=0$ differs from the others because the sample was multidomain one (it was not polarised earlier) and the application of the method described above did not give the whole value of the spontaneous polarisation. The next measurements were performed on the polarised samples. The obtained data confirmed the predicted ones resulting from the thermodynamic theory. The electric field causes the change of the temperature dependence of the polarisation, the influence is the bigger the closer the temperature is to the phase transition point. In all cases the electric field causes a diffusion of the phase transition which is greater when the intensity of the electric field increases. The values of polarisation at temperature $290 \mathrm{~K}$ obtained from the experiment are in good agreement with those obtained theoretically. The character of the $P_{\mathrm{s}}$ changes with temperature and electric field intensity is the same both for the theoretical and experimental dependencies.

The experimental results of the pyroelectric modulus are depicted in Fig. 5 . The values of the pyroelectric modulus were calculated as a temperature derivative 

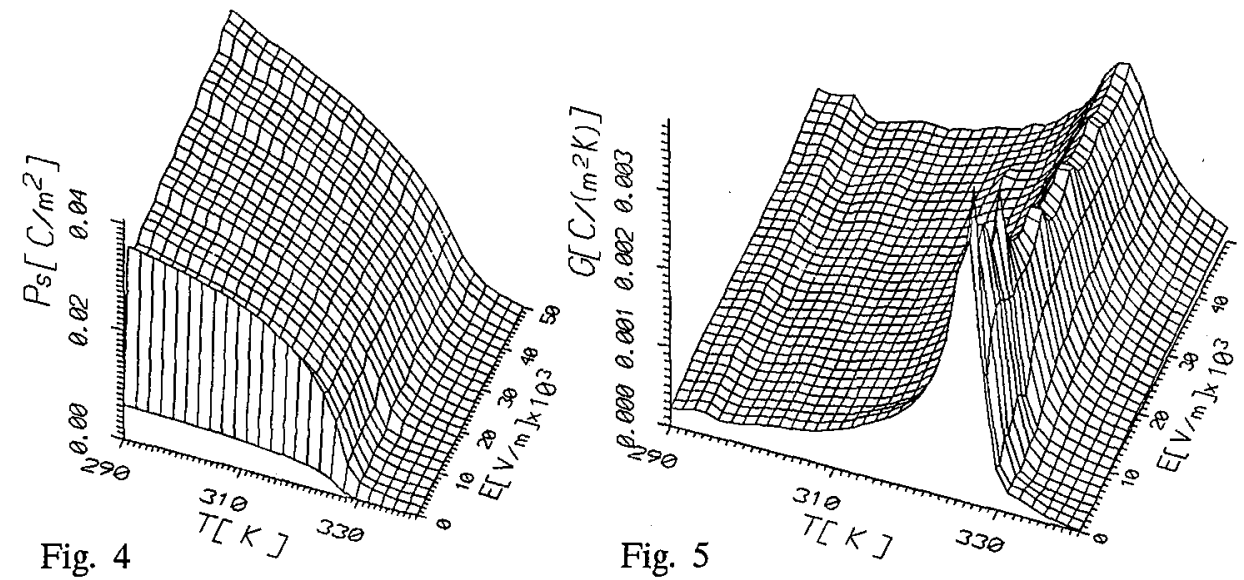

Fig. 4. Temperature dependence of the spontaneous polarisation for the TGS crystals doped with the $\mathrm{Cr}^{3+}$ chromium ions at concentration of $0.5 \%$ at different electric field intensities.

Fig. 5. Temperature dependence of pyroelectric modulus for the TGS crystals doped with the $\mathrm{Cr}^{3+}$ chromium ions at concentration of $0.5 \%$ at different electric field intensities.

of the $P_{\mathrm{s}}$. Since the $P_{\mathrm{s}}=f(T)$ results obtained at $E=0$ are different from the others due to the reasons mentioned above it is also reflected in the values of the pyroelectric modulus $(\gamma=f(T)$ at $E=0)$. From these measurements for the TGS crystals doped with the $\mathrm{Cr}^{3+}$ chromium ions it results that the pyroelectric modulus does not depend on the electric field at the temperature far from the phase transition point. On the other hand, the remarkable influence of electric field on $\gamma_{\max }$ (the maximum value of the pyroelectric modulus in the vicinity of the phase transition temperature) as found. The polarising field changes the range of the discontinuity of the pyroelectric modulus. This region undergoes broadening toward higher temperature with the increase in electric field. After crossing the Curie point the pyroelectric modulus is not rapidly dropping to the zero value but only gradually diminishes (in proportion as in the crystal the paraelectric mass significantly exceeds the ferroelectric one). $\gamma_{\max }$ decreases with an increase in electric field and simultaneously the half-width of the $\gamma=f(T)$ curve increases.

We should pay attention to a periodic fade of the pyroelectric modulus as a function of the electric field intensity.

Qualitative and quantitative character of the changes of pyroelectric modulus with temperature and electric field obtained from the experiment and calculated theoretically are in good agreement (with the exception of the $\gamma=f(T)$ dependence at $E=0$ ). At the temperature of $290 \mathrm{~K}$ the $P_{\mathrm{s}}$ value for the TGS crystals doped with the $\mathrm{Cr}^{3+}$ chromium ions are slightly larger than those for crystals doped with 2-aminopyridine and those for the pure TGS crystals. The $P_{\mathrm{s}}$ values are equal to $3.41 \times 10^{-2} \mathrm{C} / \mathrm{m}^{2}, 2.81 \times 10^{-2} \mathrm{C} / \mathrm{m}^{2}$ and $3.1 \times 10^{2} \mathrm{C} / \mathrm{m}^{2}$ for TGS crystals doped with the $\mathrm{Cr}^{3+}$ chromium ions, for crystals doped with 2-aminopyridine and for the pure TGS crystals respectively (Fig. 6). 


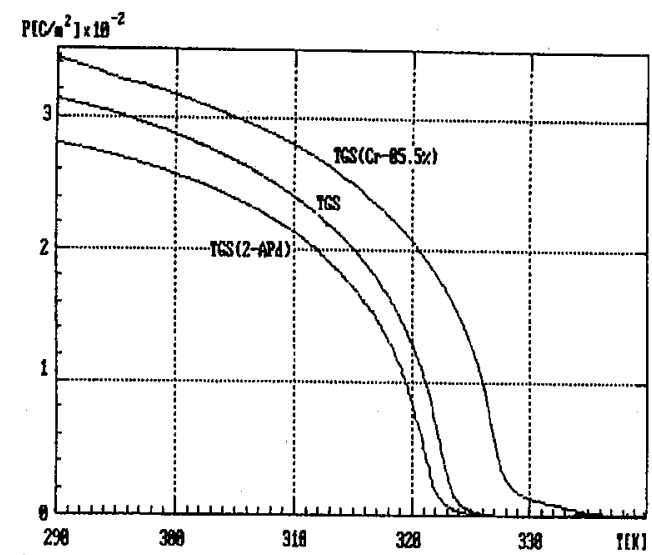

Fig. 6

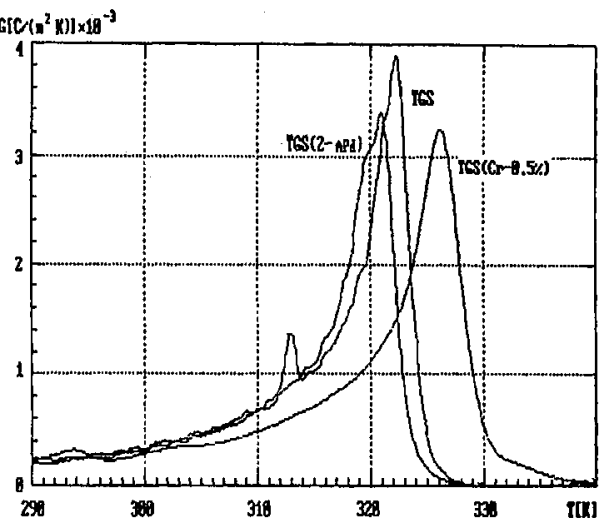

Fig. 7

Fig. 6. Temperature dependence of the spontaneous polarisation for the pure TGS crystals and those doped with 2-aminopyridine and the $\mathrm{Cr}^{3+}$ chromium ions.

Fig. 7. Temperature dependence of the pyroelectric modulus for the pure TGS crystals and those doped with 2-aminopyridine and the $\mathrm{Cr}^{3+}$ chromium ions.

At the temperature of $290 \mathrm{~K}$ the $\gamma$ values are equal to $1.78 \times 10^{-4} \mathrm{C} /\left(\mathrm{m}^{2} \mathrm{~K}\right)$, $1.98 \times 10^{-4} \mathrm{C} /\left(\mathrm{m}^{2} \mathrm{~K}\right)$ and $1.55 \times 10^{-4} \mathrm{C} /\left(\mathrm{m}^{2} \mathrm{~K}\right)$ for non-doped, doped with the $\mathrm{Cr}^{3+}$ chromium ions and 2-aminopyridine, respectively. $\gamma_{\max }$ approaches the greatest value for the non-doped crystals and the lowest one for the TGS crystals doped with the $\mathrm{Cr}^{3+}$ chromium ions (Fig. 7).

\section{Discussion}

The TGS crystals doped with the $\mathrm{Cr}^{3+}$ chromium ions presented in this paper confirmed the predicted according to the thermodynamic theory temperature dependence of spontaneous polarisation and pyroelectric modulus at different electric field intensities. The agreement is not only quantitative but also within an accepted accuracy, qualitative.

The electric field may distinguish the given direction of the lattice vibrations leading to the abandon of the principle of equipartition of energy for the particular degrees of freedom. The effect increases with increase in the electric field intensity. This causes the distortion of the statistical distribution of the lattice ion vibrations, which results in the diffusion of the phase transition and a shift of its temperature toward higher temperature. All $\gamma=f(T)$ dependencies possess the significant maxima at temperature close to the phase transition point. These maxima are related to the rapid disappearance of polarisation at the phase transition of the crystal.

These investigations did not give a convincing proof for the strict relationship between the pyroelectric modulus values in the ferroelectric phase at temperatures far from $T_{\mathrm{c}}$ and electric field intensity. On the other hand, such a relationship exists between these quantities in the close vicinity of the phase transition.

A proposal of an explanation of this fact can be presented on the base of the results given in Ref. [7] and related to the formation and breaking of weak bonding 
between chains formed by glycine II, glycine III and $\mathrm{SO}_{4}^{2-}$ groups at temperature close to $T_{\mathrm{c}}$. These bonds are responsible for existence of dipole moments of the elementary cells. Taking into account the statistical character of the formation and breaking of these bonds it may be assumed that the probability of these two phenomena are different function of temperature. At each temperature from the critical range the equilibrium between the formed and broken bonds is established and according to that the number of molecules contributing to this process is an ascending function of temperature approaching a maximum at a given temperature and then it decreases. The maximum value of this function and its half-width depend on physical properties of the crystal. The process of formation and breaking of the bonds lead to a change of the number of dipole moments in the crystal.

In the non-polarised crystal the breaking of the mentioned above bonds begins in the ferroelectric phase in the vicinity of $T_{\mathrm{c}}$. At $T_{\mathrm{c}}$ the kinetic energy of molecules is big enough to break all bonds, which leads to a disappearance of spontaneous polarisation. This fact is reflected in the fast increase in pyroelectric modulus followed by its rapid drop to zero (discontinuity at phase transition temperature). Electric field causes a temperature dispersion of the number of molecules participating in the process of formation and breaking of bonds as well as a shift of their maximum number towards higher temperatures with the increase in the electric field intensity. This fact is reflected in the behaviour of spontaneous polarisation and pyroelectric modulus (lack of discontinuity of the $\gamma=f(T)$ function at the $T_{\mathrm{c}}$ temperature).

The increase in electric field intensity leads to diminishing of the maximum value of the pyroelectric modulus and to a rise of the half-width of the $\gamma=f(T)$ function at $E=$ const. The chromium ion impurities cause the establishment of the internal electric field, which is added to the field polarising the crystal. In the particular case when the polarising electric field directed in the opposite direction to the internal field is applied, both fields are compensated. In this case many properties of the crystal, both electric and elastic, will be similar as in a non-doped crystal.

Such crystals may be regarded as two-phase systems [2]. Non-perturbed TGS crystal lattice constitutes one phase, the other - spherical domains of the crystal perturbed, which are disturbed by the doping $\mathrm{Cr}^{3+}$. Then the interaction of the electric field will be different for both phases. The electric field may cause the breaking dipoles away extrinsic films. We see it in oscillations of the pyroelectric modulus (Fig. 5).

\section{References}

[1] H. Wolniewicz, Mater. Sci. (Poland) 17, 3 (1991).

[2] T. Krajewski, J. Dubik, E. Dubik, Fiz. Diel. Rad. VIII/2, 189 (1976).

[3] S. Waplak, J. Stankowski, Acta Phys. Pol. 36, 71 (1969).

[4] R. Truell, Phys. Rev. 116, 890 (1959).

[5] C. Ying, R. Truell, J. Appl. Phys. 27, 1086 (1956).

[6] J. Malecki, Teoria fal i układów akustycznych, PWN, Warszawa 1964 (in Polish).

[7] J. Stankowski, Ferroelectrics 28, 107 (1978). 